To be submitted to Theoretical Chemistry Accounts (TCA): paper dedicated to the

UNESCO International Year of Light and Light-based Technologies (IYL 2015). TCA special Issue on Health \& Energy from the Sun: a Computational Perspective.

\title{
Describing excited states of $[n]$ cycloparaphenylenes by hybrid and double-hybrid density functionals: from isolated to weakly interacting molecules
}

J. C. Sancho-García ${ }^{1 *}$ C. Adamo ${ }^{2,3,4}$, and A. J. Pérez-Jiménez ${ }^{1}$

${ }^{1}$ Departamento de Química Física, Universidad de Alicante, 03080 Alicante, Spain

${ }^{2}$ Institut de Recherche de Chimie Paris, IRCP CNRS UMR-8247, Chimie ParisTech, École Nationale Superieure de Chimie de Paris, 11 rue P. et M. Curie, F-75231 Paris Cedex 05, France

${ }^{3}$ Institut Universitaire de France, 103 Boulevard Saint Michel, F-75005 Paris, France

${ }^{4}$ Istituto Italiano di Tecnologia, CompuNet, I-16163 Genova, Italy

November 12, 2015

*E-mail: jc.sancho@ua.es 


\begin{abstract}
We theoretically study the excited-state properties of Cycloparaphenylenes ([n]CPPs) for a deeper understanding of their photochemical properties with increasing size $n$, being $n=6-12$ the number of repeat units forming the nanoring. We apply hybrid (e.g. PBE0 and PBE0-1/3), double-hybrid (e.g. PBE0-DH and PBE-QIDH) and range-separated (e.g. $\omega$ B97X) functionals within a Time-Dependent (Tamm-Dancoff) Density Functional Theory framework. For the [6]CPP case, we concomitantly address changes in the set of selected excitedstates when going from an isolated molecule to a pair of self-assembled molecules, employing for that a dimer of parallel-like or tubular-like molecules according to the solid-state supramolecular organization found in real samples. These nanorings challenge standard theoretical methods due to the compromise needed between medium-range intra-molecular (in isolated molecules) and long-range inter-molecular (for weakly interacting pairs of molecules in condensed phases) interactions.
\end{abstract}

Key words: [n]Cycloparaphenylenes, (Time-Dependent) Density Functional Theory, Excited States, Self-aggregated Molecules. 


\section{Introduction}

The Sun emits light essentially in the visible part of the spectrum, that is, within the range of the electromagnetic field visible to the human eye, thus comprising photon energies ranging roughly from 1.6 (red) to 3.3 (violet) $\mathrm{eV}$, and in the near-infrared region at lower energies. When this radiation interacts with an organic molecule, the energy is absorbed and thereby an excited-state of a defined multiplicity is created. The spin of any state is given by the total spin of all electrons contained in the molecule, although it normally suffices to consider if all electrons are paired (i.e. a singlet state) or if some are unpaired (e.g. a triplet state). These states are key to many photoluminescence processes such as fluorescence or phosphorescence; we will thus focus in the following on the theoretical description and nature of the lowest singlet excited-states of some organic molecules of recent interest named Cycloparaphenylenes (CPPs) $[1,2]$.

The large interest in the molecules tackled here as model systems is not casual. They are formed upon bending a finite number of benzene rings until a closed structure is reached, see Figure 1, under harsh reaction conditions to overcome the associated strain energies. This synthesis has been actually accomplished for a large number of benzene units, giving rise to the family of $[n]$ Cycloparaphenylenes ( $[n] \mathrm{CPPs})$, where $n$ refers to the number of benzene units connected in para position, attracting worldwide attention [3-24]. Furthermore, since they represent the shortest sidewall fragment of armchair single-walled carbon nanotubes, these molecules are envisioned as templates for the fine-tuned synthesis of the latter through photochemically induced annealing [25]. Finally, these molecules are able to spontaneously self-assemble forming molecular crystals [26], act as nanobelts for encapsulating fullerenes 
(Saturn-like systems) [27-30], or dimerize creating nanochannels of controlled length and/or chirality [31], which might disclosure a large number of new and challenging properties upon photoexcitation, possibly bringing thus Health and/or Energy from the Sun which is the key leitmotif of the current issue.

Actually, the study of the excitonic properties of these compounds has revealed so far several interesting features. One of the first studies performed analyzed the absorption energies from the ground to the lowest singlet excited-state, as a function of the system size, compared with those of the corresponding linear paraphenylenes $[32,33]$. Interestingly, and contrarily to what it was observed for the parent linear forms, the lowest absorption energies of $[n]$ CPPs increase with the number of rings up to reach some saturation value, although very low oscillator strengths (related to absorption intensities) were always found for the cyclic forms. The absorption spectra of $[n]$ CPPs was later recorded, showing however a nearly-constant peak at around $3.65 \mathrm{eV}$ independently of system size and/or solvent effects [34]. These results were rationalized in terms of an optically forbidden singlet excited-state of lowest energy, $S_{1}$, and thus with a vanishing oscillator strength, and two degenerate (quasi-degenerate) states slightly higher in energy and dubbed as $S_{2}$ and $S_{3}$ in even (odd) nanocycles showing large oscillator strength values $[35,36]$. The corresponding lowest triplet excited-state of some of these compounds have been also investigated showing size-dependent trends similar to the $S_{1}$ case [37]. More recent studies have explored the vibronic dynamics of these excited-states [38] and the nature of associated excitons and polarons to stimulate the future design of $[n] \mathrm{CPP}$-based optoelectronic devices [39]. 
Note also that these excited states might be largely affected by environmental issues, i.e. the way in which the molecules are arranged in the trip followed from gas-phase to thin films (and then for their envisioned use in real devices) which will be also addressed here considering their associated changes in energies and/or oscillator strengths [40]. We will first study how the nature of the lowest singlet excited-state evolves with system size, employing Time-Dependent Density Functional Theory (TD-DFT), investigating next the changes when going from an isolated molecule to a weakly interacting dimer, taken at crystal structure. Due to the long-range nature of the latter interactions, we will systematically employ double-hybrid (last generation) density functionals [41], whose details are presented in the next section, together with hybrid and range-separated models, in an attempt to disentangle the subtle yet expectedly marked interplay between intra- and inter-molecular excitation energies.

\section{Theoretical details}

The functionals used here (see Table 1) can all be defined as parameterfree, and are theoretically rooted on the Adiabatic Connection Model (ACM) which defines the exchange-correlation $\left(E_{x c}\right)$ component of the total KohnSham (KS) energy as:

$$
E_{x c}[\rho]=\int_{\{\lambda=0\}}^{1} \mathcal{W}_{x c, \lambda}[\rho] d \lambda,
$$

with

$$
\mathcal{W}_{x c, \lambda}[\rho]=\left\langle\Psi_{\lambda}\left|\hat{V}_{e e}\right| \Psi_{\lambda}\right\rangle-\frac{1}{2} \iint \frac{\rho(\mathbf{r}) \rho\left(\mathbf{r}^{\prime}\right)}{\left|\mathbf{r}-\mathbf{r}^{\prime}\right|} d \mathbf{r}^{\prime} d \mathbf{r}
$$

being $\hat{V}_{e e}$ the electron-electron operator with associated mean value $\left\langle\Psi_{\lambda}\left|\hat{V}_{e e}\right| \Psi_{\lambda}\right\rangle$, and $\Psi_{\lambda}=\min _{\Psi \rightarrow \rho(\mathbf{r})}\left\langle\Psi_{\lambda}\left|\hat{T}+\lambda \hat{V}_{e e}\right| \Psi_{\lambda}\right\rangle$. A different choice of the mathe- 
matical form of $\mathcal{W}_{x c, \lambda}[\rho]$, and some associated constraints, leads to singlehybrid $[42,43]$ or double-hybrid density functionals [44-47], whose form may be finally casted in a general expression such as:

$$
E_{x c}=\lambda_{x} E_{x}^{\mathrm{EXX}}[\phi]+\left(1-\lambda_{x}\right) E_{x}[\rho]+\lambda_{c} E_{c}^{\mathrm{PT} 2}\left[\phi, \phi^{\prime}\right]+\left(1-\lambda_{c}\right) E_{c}[\rho],
$$

with the values theoretically found for $\lambda_{x}$ and for (if any) $\lambda_{c}$ given in Table 1. Note that $E_{x}^{\mathrm{EXX}}[\phi]$ and $E_{c}^{\mathrm{PT} 2}\left[\phi, \phi^{\prime}\right]$ are the exact-like exchange (EXX) and the perturbation correlation correction up to second-order (PT2), respectively, with $\phi\left(\phi^{\prime}\right)$ the occupied (unoccupied) orbitals self-consistently obtained. A (single-)hybrid functional neglects the latter term, and thus $\lambda_{c}=0$, while some modern double-hybrid functionals force the condition $\lambda_{c}=\lambda_{x}^{3}$.

Then, calculations of selected excited states, with both hybrid and doublehybrid density functionals, were done using the Tamm-Dancoff Approximation (TDA) $[48,49]$, which slightly reduces the computational cost with respect to the full Time-Dependent (TD) equations. This approach gives also accurate results for both singlet- and triplet excited-states [50] and absorption and emission vibrational band shapes [51]. Whereas for hybrid (e.g. PBE0 [52] or PBE0-1/3 [53]) density functionals the excitation energies $\Omega$ are obtained following the standard formalism, which have been thoroughly benchmarked in last years $[54,55]$, an additional correction is however needed for the case of double-hybrid (e.g. PBE0-DH or PBE-QIDH) models [56], with the form:

$$
\Omega^{\prime}=\Omega+\lambda_{c} \Delta_{(\mathrm{D})},
$$

with $\lambda_{c}$ the specific weight given in Eq. (3) to the perturbative term, and $\Delta_{(\mathrm{D})}$ its corresponding correction for excited-states [57]. Thus, these latter calculations are done in a two-step fashion: (i) $\Omega$ is first obtained with 
the resulting amplitudes and eigenvalues after converging a set of KohnSham equations using the exchange-correlation potential given by $v_{x c}[\rho]=$ $\lambda_{x} \frac{\delta E_{x}^{\mathrm{EXX}}}{\delta \rho}+\left(1-\lambda_{x}\right) \frac{\delta E_{x}[\rho]}{\delta \rho}+\left(1-\lambda_{c}\right) \frac{\delta E_{c}[\rho]}{\delta \rho}$; and (ii) based on these solutions, the $\Delta_{(\mathrm{D})}$ correction is scaled by $\lambda_{c}$ and added to give the final $\Omega^{\prime}$ of the excitation energy of each state. This scheme has been shown to behave accurately for a representative set of organic molecules [58-60].

We used in all cases the $6-31 \mathrm{G}^{*}$ basis set due to its good trade-off between accuracy and computational cost. Note that, for the [6]CPP compound chosen as a benchmark system, the excited-state energies calculated with the large def2-TZVP [61] basis set were found to differ by less than $0.1 \mathrm{eV}$, independently of the functionals selected, with respect to the values obtained with the $6-31 \mathrm{G}^{*}$ one. Furthermore, the use of a continuum model for estimating solvent effects, the COnductor-like Screening MOdel (COSMO) [62-64], allowed also to rule out its negligible influence on the final values, and thus all the calculations reported here will refer to gas-phase unless otherwise noticed.

Other technical details are also presented next: (i) the geometry of all the compounds was previously optimized at the B3LYP-D3(BJ)/6-31+G* level [65] and used rigidly herein; (ii) all the calculations were done with the ORCA 3.0.2 package [66] increasing systematically the numerical thresholds for ground- and excited-state calculations (e.g. keywords TightSCF, Grid6, NoFinalGrid); (iii) the frozen core approximation was utilized in all cases for calculating the perturbation correction; and (iv) the 'resolution-of-theidentity' (RI) and 'chain-of-spheres' (COSX) techniques [67,68] were always employed, particularly to reduce the additional computational cost associated with double-hybrid models. 


\section{Results and discussion}

\subsection{Increasingly longer $[n] \mathrm{CPPs}$}

Table 2 shows all vertical absorption energies for the three lowest singlet excited-states, calculated with the parameter-free functionals described so far, together with their corresponding oscillator strengths. Upon inspecting the values, and almost independently of the method used, we can affirm that: (i) the $S_{1}$ state (a $\pi \rightarrow \pi^{\star}$ transition dominated by a HOMO to LUMO excitation) is optically forbidden, with vanishing (very low) oscillator strengths in even (odd) $[n]$ CPPs, and systematically increasing in energy with system size; (ii) the $S_{2}$ and $S_{3}$ states (a $\pi \rightarrow \pi^{\star}$ transition dominated by a HOMO to $(\mathrm{L}+1) \mathrm{UMO} /(\mathrm{L}+2) \mathrm{UMO}$ or $(\mathrm{H}-2) \mathrm{OMO} /(\mathrm{H}-1) \mathrm{OMO}$ to $\mathrm{LUMO}$ excitations) are perfectly (almost) degenerate in even (odd) $[n]$ CPPs, keeping a nearly-constant value up to $0.1 \mathrm{eV}$ with system size, and show large enough oscillator strength values typical of an optically allowed state; and (iii) larger nanohoops are expected to absorb light more efficiently, with oscillator strengths being up to three times higher when going from [6]CPP to [12]CPP. These findings are fully consistent with the experimental information available so far. For instance, the experimental UV-vis absorption spectra of these compounds roughly overlap, independently of their size, with a highest absorption maxima found at $338-340 \mathrm{~nm}(3.64-3.67 \mathrm{eV})$ not affected by solvent effects $[69,70]$, which was formerly attributed to that allowed $S_{2} / S_{3}$ states.

Previous literature results were obtained for $[n]$ CPPs employing only hybrid (e.g. B3LYP) and/or range-separated functionals (e.g. CAM-B3LYP), 
and thus we will compare next our TDA-PBE0/6-31G*//B3LYP-D3(BJ)/6$31+\mathrm{G}^{*}$ and TDA-PBE0-1/3/6-31G*//B3LYP-D3(BJ)/6-31+G* results with those obtained before at the popular TD-B3LYP/6-31G*//B3LYP/6-31G* level $[33,34]$. In the case of the $S_{1}$ state, our results are consistently found roughly above $0.2-0.4 \mathrm{eV}$ from those obtained at the latter level, which might be preliminarily attributed to the slightly larger value of $\lambda_{x}$ in PBE0 $\left(\lambda_{x}=1 / 4\right)$ or PBE0-1/3 $\left(\lambda_{x}=1 / 3\right)$ with respect to B3LYP $\left(\lambda_{x}=0.20\right)$. Note that we have previously isolated the negligible difference between TDAPBE0 and full TD-PBE0, or between the TDA-B3LYP and the TD-B3LYP calculations, the latter without neglecting the $\mathbf{B}$ deexcitation coupling term, and found it as small as $0.01-0.04 \mathrm{eV}$ for the set of states reported in Table 2 for the [6]CPP molecule. Nonetheless, all these mentioned model chemistries (i.e. PBE0, PBE0-1/3 or B3LYP) are consistently accurate, predicting an absorption energy for the $S_{2}=S_{3}$ singlet excited-states rather close to the experimental, and nearly-constant, value of $3.67 \mathrm{eV}$. However, we have also observed the appearance of some intruder states for the smaller nanohoops when these hybrid PBE0 and PBE0-1/3 models are used. For instance, taking the [6]CPP case as example, a bright and degenerate $S_{5} / S_{6}\left(S_{4} / S_{5}\right)$ state is predicted with the former (latter) model. This anomaly is cured when a double-hybrid model (e.g. PBE0-DH or PBE-QIDH) is used, showing a robust performance and high oscillator strength values for all system sizes, although admittedly, the vertical $S_{2} / S_{3}$ absorption energies computed by these double-hybrid functionals are overestimated with respect to the experimental value, by up to $0.4-0.6 \mathrm{eV}$. 


\subsection{Benchmark study of isolated [6]CPP}

To further understand in depth the (somehow unexpected) behavior of double-hybrid functionals, we take the [6]CPP case as example and first investigate how the vertical absorption energies of the lowest singlet excitedstates evolve as a function of the $\lambda_{x}$ value. Figure 2 displays the results obtained when the sequence PBE0 $\left(\lambda_{x}=1 / 4\right)$, PBE0-1/3 $\left(\lambda_{x}=1 / 3\right)$, PBE0$\mathrm{DH}\left(\lambda_{x}=1 / 2\right)$ and PBE-QIDH $\left(\lambda_{x}=3^{-1 / 3}\right)$ is considered. The dashed line shows the values of $\Omega^{\prime}$ obtained according to Eq. (4). We can readily see how the absorption energies for either the $S_{1}$ or the $S_{2} / S_{3}$ state are mostly driven by the values of $\lambda_{x}$. For the double-hybrid cases, the size of the $\lambda_{c} \Delta_{(\mathrm{D})}$ correction, which amounts to roughly up to $-0.2 \mathrm{eV}$, does not entirely compensate that overestimation suffered, which renders the PBE0 values the closest to the experimental estimate. This trend is confirmed also for the family of parameterized B3LYP $\left(\lambda_{x}=0.2\right)$, B2-PLYP $\left(\lambda_{x}=0.53\right)$ and B2GP-PLYP $\left(\lambda_{x}=0.65\right)$ models.

This overestimation found by double-hybrid functionals has been also reported for range-separated functionals (e.g. CAM-B3LYP) previously used on these systems $[36,39]$. In order to further investigate this point, we have also applied one of the most recent models, namely the $\omega$ B97X functional $[71,72]$, which might help to shed some light on the performance of the different methods assessed through this work. Note that the closely related $\omega$ B97X-D functional behaves similarly to the latter model, with differences in excitation energies being less that $0.1 \mathrm{eV}$ for all the states considered. We can see in Table 2 how this method also largely overestimates the experimental absorption maxima in $[n]$ CPPs, as well as it does a closely related (LC-BLYP) range-separated version [73] also tested here, yet providing the 
correct trend for oscillator strength values. Note that recent investigations have also shown some differences between oscillator strengths depending on the functional chosen, with range-separated models behaving more accurately than standard hybrid functionals compared to EOM-CCSD benchmark values [74].

In an effort to better understand such oscillator strength differences, we depict in Figure 3 the transition densities calculated by TDA-PBE0 and TDA- $\omega$ B97X methods for the three lowest singlet excited-states of the [6]CPP molecule. This quantity is known to represent changes in the electronic density upon excitation to a selected excited-state. For the $S_{1}$ state both methods predict a vanishing transition dipole moment $\vec{\mu}_{10}$ (where the subindex refers to the transition from the fundamental to the lowest singlet excitedstate) due to the fact that in such a circular geometry all the elementary transition dipole moments (localized between pairs of atoms following a headto-tail pattern) will effectively cancel, giving rise to a null vectorial sum. On the other hand, only the TDA- $\omega$ B97X calculations gave a sufficiently distorted distribution of the orbital contributions to the transition density for excited-states $S_{2}$ and $S_{3}$, and thus $\vec{\mu}_{20} / \vec{\mu}_{30} \neq 0$. This pronounced asymmetry leads in fact to the constructive superposition of the set of localized transition dipole moments, and then contributing to a net oscillator strength $(f$ of the ith excited-state is proportional to $\left.\left|\vec{\mu}_{i 0}\right|^{2}\right)$. This picture is fully consistent with the oscillator strength values reported in Table 2, and holds for double-hybrid functionals too, indicating the importance of correctly dealing with intra-molecular long-range interactions for the adequate representation of the excited-state topology and orbital distribution. 
In order to better rationalize the assignments of UV-vis absorption energies, we consider next the Natural Transition Orbitals (NTO) $[75,76]$. In this way, the transition density matrix coupling the ground-state $|\Psi\rangle$ and the excited-state $\left|\Psi^{\star}\right\rangle$ wavefunctions is made diagonal, and then each hole in the occupied space is associated to a single particle in the virtual space, without changing the corresponding excitation energies obtained here. This tool allows an easier (more compact) representation of the orbital transitions leading to the lowest singlet excited-state (see Table 3 for the [6]CPP case). We can see how the $S_{1}$ state arises from a transition from the Highest Occupied Natural Transition Orbital (HONTO) to the Lowest Unoccupied Natural Transition Orbital (LUNTO), whereas the $S_{2}$ and $S_{3}$ states are found degenerate and arise from a HONTO to LUNTO and (H-1)ONTO to $(\mathrm{L}+1) \mathrm{UNTO}$ mix of excitations. These frontier (and fully delocalized along the nanoring) NTO are depicted in Figure 4, with the transitions assigned to be of a $\pi \rightarrow \pi^{\star}$ nature according to the nature of the orbitals involved.

These set of NTO involved in the transition(s) leading to the selected excited-states can be also used to estimate its nature (i.e. localized or chargetransfer) thanks to the following index recently proposed [77]:

$$
\Delta \mathbf{r}=\frac{\sum_{i a} \kappa_{i a}^{2}\left|\left\langle\phi_{a}^{\prime}|\hat{\mathbf{r}}| \phi_{a}^{\prime}\right\rangle-\left\langle\phi_{i}^{\prime}|\hat{\mathbf{r}}| \phi_{i}^{\prime}\right\rangle\right|}{\sum_{i a} \kappa_{i a}^{2}},
$$

with $\kappa_{i a}$ the contribution of a particular occupied-virtual $(i a)$ orbital pair to the excitation, and $\left\langle\phi_{i}^{\prime}|\hat{\mathbf{r}}| \phi_{i}^{\prime}\right\rangle$ the orbital centroid. The use of some metrics to characterize the nature of an excited-state has revealed recently as a highly useful tool [78-83], and actually a threshold for $\Delta \mathbf{r}$ of $1.5-2.0 \AA$ is proposed as a sign of having a (marked) charge-transfer excitation or not [84]. This is easily understood if one considers the $\Delta \mathbf{r}$ value as a kind of exciton free path. Our calculations estimate a value of $\Delta \mathbf{r}\left(S_{1}\right)=0.00 \AA$ and 
$\Delta \mathbf{r}\left(S_{2}=S_{3}\right)=0.01 \AA$ for an isolated [6] CPP molecule, irrespective of the set of NTO used, derived either from TDA-PBE0 or from TDA-PBE0-DH calculations. This rules out any charge-transfer excitation and helps to understand the accuracy for excitation energies of the TDA-PBE0 calculations performed, since for non-charge-transfer (i.e. standard) excitations this functional is known to be a highly accurate method $[85,86]$.

\subsection{Interacting dimers: the [6] CPP case}

The crystal packing of [6]CPP is particularly interesting yet challenging. These molecules self-assemble in the solid-state following the pattern included in Figure 5, where a pair of interacting molecules belonging to the same (the $a b$ plane) or to different slabs (along the $c$ axis) is shown, and contrarily to the rest of $[n] \mathrm{CPP}$ systems where a herringbone-like arrangement is experimentally found [15]. The geometries displayed were previously optimized at the BLYP-D3(BJ)/def2-TZVP level [65] and used without further modifications here for the TDA-DFT reported calculations. When the two molecules superimpose in a tubular-like configuration, see Figure 5, their dispersioncorrected interacting energy was calculated to be nearly twice the energy of the parallel molecules, which might anticipate their growing tubular-like mechanism and a chemical path to control their annealing into nanotubes of that diameter and configuration.

This supramolecular organization might consequently alter the electronic excitations when passing from an isolated single molecule to a pair of weakly bound interacting ones [87]; we will thus consider next what happens when any of these two unique pairs become excited. Be $\left|\Psi_{a}^{\star}\right\rangle\left(\left|\Psi_{b}^{\star}\right\rangle\right)$ the wave- 
function of an isolated molecule dubbed as $a$ (or $b$ ) in its excited-state, and $\left|\Psi_{a} \Psi_{b}^{\star}\right\rangle$ or $\left.\left|\Psi_{a}^{\star} \Psi_{b}\right\rangle\right)$ the corresponding excited-state when the dimer is formed. If both molecules behave identically, the excited-state combines both situations, and then the total wavefunction is $|\Psi\rangle=\frac{1}{\sqrt{2}}\left(\left|\Psi_{a} \Psi_{b}^{\star}\right\rangle \pm\left|\Psi_{a}^{\star} \Psi_{b}\right\rangle\right)$. The dimer Hamiltonian can be written as $\hat{H}=\hat{H}_{a}+\hat{H}_{b}+\hat{V}$, with $\hat{H}_{i}$ corresponding to the isolated molecules and $\hat{V}$ containing the weak interactions between both units. Inserting the total wavefunction to solve the associated Schrödinger equation for the dimer leads to the splitting of the excited-state energy into two levels, $\Omega_{\text {dimer }}^{\star}=\Omega_{i}^{\star} \pm \beta$, with $\Omega_{i}^{\star}$ the $i$ th. excited-state energy of the isolated constituting molecules and $\beta$ the resonance interaction energy $\left\langle\Psi_{a}^{\star} \Psi_{b}|\hat{V}| \Psi_{a} \Psi_{b}^{\star}\right\rangle$. The quantity $\beta$ depends on the relative orientation and distance between the monomers of the weakly bound (physical) dimer, and can be thus driven by long-range interactions and vary for each excited-state. The following scheme aims at illustrating the (perfect) electronic coupling between two monomers, neglecting polarization effects in the rest of this work, showing how there always should be two split levels separated by $2 \beta$ :

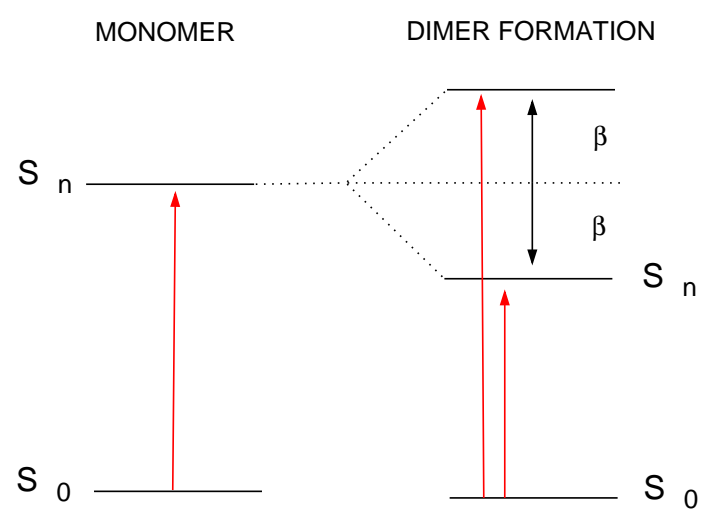

Scheme 1

Furthermore, in the [6]CPP case analyzed in detail here, the degeneracy 
of the frontier orbitals leading to the lowest singlet excited-states, dubbed before as $S_{1}-S_{3}$, manifests in a manifold of nearly-degenerate orbitals when the dimer state is formed. Looking for instance at the PBE0/6-31G* window of relevant orbitals for the transitions in the monomer, i.e. from the ( $\mathrm{H}-2) \mathrm{OMO}$ to the $(\mathrm{L}+2) \mathrm{UMO}$, their eigenenergies (in $\mathrm{eV}$ ) are $-6.07,-6.07$, $-5.18,-1.70,-0.84$, and -0.84 , with the next occupied or virtual molecular orbitals lying well separated in energy, by roughly $1 \mathrm{eV}$, from these values. The energies (in $\mathrm{eV}$ ) of the corresponding orbitals in the dimer (for the tubular-like configuration taken as example) become now $-6.17,-6.16$, $-6.16,-6.15,-5.26,-5.24,-1.89,-1.88,-1.02,-1.01,-0.97$, and -0.97 , without influencing too much the HOMO-LUMO gap, thus constituting this set of orbitals the active space for the lowest singlet excited-states created upon the dimer formation.

Table 4 presents the energy and oscillator strengths of the singlet excitedstates created in the dimer configurations tackled, thus upon the parallel- or tubular-like aggregation of two single molecules. We will restrict in the following, to avoid excessive information, to a subset only of all the formed excited-states. Starting with the TDA-PBE0/6-31G* results, we can observe how the states are now grouped in nearly-degenerate pairs, with values (in $\mathrm{eV}$ ) of $2 \beta$ being 0.4 (0.3) for the lowest excited-states in the parallellike (tubular-like) dimer (i.e. compare values of $\Omega\left(S_{1}\right)$ in Table 2 with the corresponding $\Omega\left(S_{1}\right) / \Omega\left(S_{2}\right)$ and $\Omega\left(S_{3}\right) / \Omega\left(S_{4}\right)$ excitation energies gathered in Table 4). Then, if we inspect the following manifold of excited-states upon the dimer formation, values of $2 \beta$ are correspondingly 0.2 and $0.1 \mathrm{eV}$. However, we underline again how the TDA-PBE0 level of theory is unable to predict large oscillator strength values for any of the lowest excited-states 
reported in Table 4.

Therefore, values of $2 \beta$ (qualitatively speaking) are always higher for the parallel-like than for the tubular-like arrangement, which might be attributed to the excimer (excimer $=$ excited dimer) formation in the case of the former, which are always characterized by stronger resonance energies and are commonly found in $\pi-\pi$ interacting chromophores. Note also that the specific through-space interactions existing for a tubular-like dimer resemble those found in a herringbone-like arrangement, but those driven the parallel-like dimer are those commonly found in stacked and flat $\pi$-conjugated molecules and are stronger and more prone to excimer formation. These excited molecular complexes demands the most from quantum-chemical methods [88], and we will thus rely in the following in the use of double-hybrid or range-separated functionals for the adequate modelling of excited-states in dimers.

Actually, we remind that the oscillator strength value of an ith excitedstate is proportional to the modulus of the corresponding transition dipole moment, $f \propto\left|\vec{\mu}_{i 0}\right|^{2}$, where $\mu_{i 0}=\left\langle\Psi_{0}|\hat{\mu}| \Psi_{i}^{\star}\right\rangle$, which for a dimer formation translates into the expression $\left\langle\Psi_{a} \Psi_{b}|\hat{\mu}| \frac{1}{\sqrt{2}}\left(\Psi_{a} \Psi_{b}^{\star} \pm \Psi_{a}^{\star} \Psi_{b}\right)\right\rangle$ according to the definition of the dimer excited-state described above. Thus, the orientation and strength of the total transition dipole moment will depend on how those of the individual molecules will align, or in other words in the relative orientation of the molecules constituting the dimer, and may be constructive or destructive, leading thus to a bright or dark state respectively [89]. We can distinguish between $\mathrm{H}$ - and J-aggregates, the former (latter) refers to sideby-side (head-to-tail) interactions between the transition dipole moments of 
the individual molecules. Consequently, H-aggregates (J-aggregates) lead to an hypsochromic or blue-shift (bathochromic or red-shift) absorption spec$\operatorname{tra}[90]$.

Starting from the tubular-like dimer, the absorption energy for those states showing a large oscillator strength can not be distinguished from that of the parent molecule (i.e. compare values of $\Omega^{\prime}\left(S_{2}\right) / \Omega^{\prime}\left(S_{3}\right)$ in Table 2 with the corresponding $\Omega^{\prime}\left(S_{7}\right) / \Omega^{\prime}\left(S_{8}\right)$, for the PBE0-DH and PBE-QIDH cases, or $\Omega^{\prime}\left(S_{5}\right) / \Omega^{\prime}\left(S_{6}\right)$ for the $\omega$ B97X case in Table 4) indicating thus an almost vanishing resonance energy $(\beta \approx 0)$ or, in other words, a very weak excitonic coupling. This is the typical case for large intermolecular distances, as it happens for this configuration with the closest $\mathrm{C}-\mathrm{C}$ distance between both molecules being above $3.9 \AA$, and low $\pi-\pi$ overlap between the constituent units. Contrarily to this picture, the closest $\mathrm{C}-\mathrm{C}$ distance between the two molecules forming the parallel-like dimer amounts to only $3.3 \AA$, with energies being consistently red-shifted by $0.17-0.19 \mathrm{eV}$ with respect to that of the isolated monomer, a typical feature of J-type interactions having some well-known consequences for the absorption and emission spectra such as high radiative rates or superluminiscence $[91,92]$.

The analysis of the $\Delta \mathbf{r}$ values might be also employed to disclose if some of these excitations can be assigned to be of a charge-transfer nature, stabilizing thus the corresponding excimer. Figure 6 shows how this is indeed the case for the states numbered as $S_{3}$ and $S_{4}$, in both parallel-like and tubular-like configurations, largely exceeding the values of the calculated $\Delta \mathbf{r}$ the threshold of $1.5-2.0 \AA$ previously proposed as a cutoff for confirming a (marked) charge-transfer excitation [84]. These charge-transfer states can be 
viewed as those having an electron in one molecule and a hole on the other, and are always difficult to describe by routine TD-DFT calculations [93]. To further illustrate this issue, Figure 7 displays the set of NTO involved in the excitations leading to the $S_{1}$ and $S_{3}$ excited-states, chosen as examples of standard and charge-transfer excitations, respectively, and for the parallel-like dimer. According to the notation previously employed for these orbitals, $\kappa_{i a}\left[\phi_{i}^{\prime} \rightarrow \phi_{a}^{\prime}\right]$, where $\kappa_{i a}$ is the contribution of a particular occupiedvirtual orbital pair to the excitation, we can observe that: (i) the $S_{1}$ state is mostly characterized by a mix of excitations 0.538 [HONTO $\rightarrow$ LUNTO] and 0.385 [(H-1)ONTO $\rightarrow(\mathrm{L}+1) \mathrm{UNTO}]$, whose orbitals for both transitions are distributed along the whole dimer; and (ii) the $S_{3}$ state is characterized by a mix of excitations $0.650[\mathrm{HONTO} \rightarrow \mathrm{LUNTO}]$ and $0.342[(\mathrm{H}-1) \mathrm{ONTO} \rightarrow(\mathrm{L}+1) \mathrm{UNTO}]$, whose orbitals are now localized in different molecules of the dimer, constituting thus a clear example of a charge-transfer excitation.

\section{Concluding remarks}

We have first reported how optical properties of $[n] \mathrm{CPP}$ compounds $(n=6-12)$ evolve with system size, employing for that a new set of novel density functionals (i.e. PBE0-DH, PBE-QIDH or $\omega$ B97X) together with more standard (e.g. PBE0 or PBE0-1/3) approximations. Although vertical absorption energies are accurately reproduced by the latter methods, the spatial distribution of orbitals and associated transition densities is better described by the former and more modern models. Thus, the particular chemical structure of these nanorings forces the use of methods able to accurately deal with both medium- and long-range intra-molecular interactions. 
In a further step, we have analyzed the different molecular complexes found after self-aggregation of a pair of [6]CPP molecules, which offers a unique opportunity to understand structure-property relationships in real samples. We have herein studied two structural configurations (i.e. packing motifs) to identify and characterize in advance the optical signatures and nature of the involved excited-states in solid-state samples. The interplay between the way in which these molecules spontaneously self-assemble, following a very directional and dense pattern, and their response upon photoexcitation to become efficient fluorophores, might lead to the generation of some specific optical and/or photophysical functionality needed to be spectroscopically confirmed.

In summary, the modern computational tools employed along this work allow to establish a firm connection between the properties of isolated and aggregated molecules, thanks to the use in all cases of double-hybrid and/or range-separated functionals able to incorporate naturally all kind of interactions, both medium- and long-range ones, needed to adequately switch from intra- to inter-molecular properties in a balanced way. We hope to have concomitantly provided valuable insights to motivate further experimental studies with these appealing molecules.

\section{Acknowledgements}

This work is supported by the "Ministerio de Economía y Competitividad" of Spain and the "European Regional Development Fund" through project CTQ2014-55073-P. 


\section{References}

[1] Golder MR, Jasti R (2015) Acc. Chem. Res. 48: 557.

[2] Lewis SE (2015) Chem. Soc. Rev. 44: 2221.

[3] Jasti R, Bhattacharjee J, Neaton JN, Bertozzi CR (2008) J. Am. Chem. Soc. 130: 17646.

[4] Jasti R, Bertozzi CR (2010) Chem. Phys. Lett. 494: 1.

[5] Yamago S, Watanabe Y, Iwamoto T (2011) Angew. Chem. Int. Ed. 50: 3244 .

[6] Segawa Y, Omachi H, Itami K (2010) Org. Lett. 12: 2262.

[7] Bachrach S, Stück D (2010) J. Org. Chem. 75: 6595.

[8] Iwamoto T, Watanabe Y, Sakamoto Y, Suzuki T, Yamago S (2011) J. Am. Chem. Soc. 133: 8354.

[9] Sisto TJ, Golder MR, Hirst ES, Jasti R (2011) J. Am. Chem. Soc. 133: 15800.

[10] Hitosugi S, Nakanishi W, Yamasaki T, Isobe H (2011) Nat. Commun. 2: 492.

[11] Li HB, Page AJ, Irle S, Morokuma K (2012) ChemPhysChem 13: 1479.

[12] Sisto TJ, Xian X, Jasti R (2012) J. Org. Chem. 77: 5857.

[13] Darzi ER, Sisto TJ, Jasti R (2012) J. Org. Chem. 77: 6624.

[14] Itami K (2012) Pure Appl. Chem. 84: 907.

[15] Xia J, Jasti R (2012) Angew. Chem. Int. Ed. 51: 2474. 
[16] Kayahara E, Iwamoto T, Suzuki T, Yamago S (2013) Chem. Lett. 42: 621.

[17] Omachi H, Nakayama T, Takahashi E, Segawa Y, Itami K (2013) Nat. Chem. 5: 572 .

[18] Fujitsuka M, Tojo S, Iwamoto T, Kayahara E, Yamago S, Majima T (2014) J. Phys. Chem. Lett. 5: 2302.

[19] Kayahara E, Patel VK, Yamago S (2014) J. Am. Chem. Soc. 136: 2284.

[20] Yamago S, Kayahara E, Iwamoto T (2014) Chem. Rec. 14: 84.

[21] Evans PE, Jasti R (2014) Top. Curr. Chem. 349: 249.

[22] Evans PE, Darzi ER, Jasti R (2014) Nat. Chem. 6: 404.

[23] Alvarez MP, Burrezo PM, Kertesz M, Yamago S, Xia J, Jasti R, López Navarrete JT, Taravillo M, Baonza VG, Casado J (2014) Angew. Chem. Int. Ed. 53: 7033.

[24] Toriumi N, Muranaka A, Kayahara E, Yamago S, Uchiyama M (2015) J. Am. Chem. Soc. 137: 82.

[25] Hirst ES, Jasti R (2012) J. Org. Chem. 77: 10473.

[26] Xia J, Bacon JW, Jasti R (2012) Chem. Sci. 3: 3018.

[27] Iwamoto T, Watanabe Y, Sadahiro T, Haino T, Yamago S (2011) Angew. Chem. Int. Ed. 50: 8342.

[28] Iwamoto T, Watanabe Y, Takaya H, Haino T, Yasuda N, Yamago S (2013) Chem. Eur. J. 19: 14061. 
[29] Iwamoto T, Slanina Z, Mizorogi N, Guo J, Akasaka T, Nagase S, Takaya H, Yasuda N, Kato T, Yamago S (2014) Chem. Eur. J. 20: 14403.

[30] Rehman HU, McKee NA, McKee ML (2015) J. Comput. Chem. DOI:10.1002/jcc.23979

[31] Xia J, Golder MR, Foster ME, Wong BM, Jasti R (2012) J. Am. Chem. Soc. 134: 19709.

[32] Wong BM (2009) J. Phys. Chem. C 113: 21921.

[33] Wong BM, Lee JW (2011) J. Phys. Chem. Lett. 2: 2702.

[34] Segawa Y, Fukuzawa A, Matsuura S, Omachi H, Yamaguchi S, Irle S, Itami K (2012) Org. Biomol. Chem. 10: 5979.

[35] Camacho C, Niehaus TA, Itami K, Irle S (2013) Chem. Sci. 4: 187.

[36] Adamska L, Nayyar I, Chen H, Swan AK, Oldani N, Fernández-Alberti N, Golder MR, Jasti R, Doorn SK, Tretiak S (2014) Nano Lett. 14: 6539 .

[37] Hines DA, Darzi ER, Jasti R, Kamat PV (2014) J. Phys. Chem. A 118: 1595.

[38] Reddy VS, Camacho C, Xia J, Jasti R, Irle S (2014) J. Chem. Theory Comput. 10: 4025.

[39] Liu J, Adamska L, Doorn SK, Tretiak S (2015) Phys. Chem. Chem. Phys. 17: 14613.

[40] Köhler A, Bässler H, Electronic Processes in Organic Semiconductors, Wiley-VCH, Weinheim, 2015. 
[41] Sancho-García JC, Adamo C (2013) Phys. Chem. Chem. Phys. 15: 14581.

[42] Perdew JP, Ernzenhof M, Burke K (1996) J. Chem. Phys. 105: 9982.

[43] Cortona P (2012) J. Chem. Phys. 136: 086101.

[44] Sharkas A, Toulouse J, Savin A (2011) J. Chem. Phys. 134: 064113.

[45] Brémond E, Adamo C (2011) J. Chem. Phys. 135: 024106.

[46] Toulouse J, Sharkas K, Brémond E, Adamo C (2011) J. Chem. Phys. 135: 101102 .

[47] Brémond E, Sancho-García JC, Pérez-Jiménez AJ, Adamo C (2014) J. Chem. Phys. 141: 031101.

[48] Grimme S (1996) Chem. Phys. Lett. 259: 128.

[49] Hirata S, Head-Gordon M (1999) Chem. Phys. Lett. 314: 291.

[50] Moral M, Muccioli L, Son WJ, Olivier Y, Sancho-García JC (2015) J. Chem. Theory Comput. 11: 168.

[51] Chantzis A, Laurent AD, Adamo C, Jacquemin D (2013) J. Chem. Theory Comput. 9: 4517.

[52] Adamo C, Barone V (1999) J. Chem. Phys. 110: 6158.

[53] Guido C, Brémond E, Adamo C, Cortona P (2013) J. Chem. Phys. 138: 021104.

[54] Moore B, Charaf-Eddim A, Planchat A, Adamo C, Autschbach J, Jacquemin D (2014) J. Chem. Theory Comput. 10: 4599. 
[55] Jacquemin D, Adamo C (2015) Top. Curr. Chem. 368: 347.

[56] Grimme S, Neese F (2007) J. Chem. Phys. 127: 154116.

[57] Head-Gordon H, Rico RJ, Oumi M, Lee TJ (1994) Chem. Phys. Lett. 219: 21.

[58] Goerigk L, Moellmann J, Grimme S (2009) Phys. Chem. Chem. Phys. 11: 4611.

[59] Goerigk L, Grimme S (2010) J. Chem. Phys. 132: 184103.

[60] Di Meo F, Trouillas P, Adamo C, Sancho-García JC (2013) J. Chem. Phys. 139: 164104.

[61] Weigend F, Ahlrichs R (2005) Phys. Chem. Chem. Phys. 7: 3297.

[62] Klamt A, Schürmann G (1993) J. Chem. Soc. Perkin Trans. 2: 799.

[63] Andzelm J, Kömel C, Klamt A (1995) J. Chem. Phys. 103: 9312.

[64] Sinnecker S, Rajendran A, Klamt A, Diedenhofen M, Neese F (2006) J. Phys. Chem A 110: 2235

[65] Climent-Medina JV, Pérez-Jiménez AJ, Moral M, San-Fabián E, Sancho-García JC (2015) ChemPhysChem 16: 1520.

[66] Neese F (2012) WIREs Comput. Mol. Sci. 2: 73.

[67] Neese F (2003) J. Comp. Chem. 24: 1740.

[68] Neese F, Wennmohs A, Hansen A, Becker U (2009) Chem. Phys. 356: 98.

[69] Iwamoto T, Watanabe Y, Sakamoto Y, Suzuki T, Yamago S (2011) J. Am. Chem. Soc. 133: 8354. 
[70] Fujitsuka M, Cho DW, Iwamoto T, Yamago S, Majima T (2012) Phys. Chem. Chem. Phys. 14: 14585.

[71] Chai JD, Head-Gordon M (2008) J. Chem. Phys. 128: 084106.

[72] Chai JD, Head-Gordon M (2008) Phys. Chem. Chem. Phys. 10: 6615.

[73] Iikura H, Tsuneda T, Yanai T, Hirao K (2001) J. Chem. Phys. 115: 3540 .

[74] Caricato M, Trucks GW, Frisch MJ, Wiber KB (2011) J. Chem. Theory Comput. 7: 456.

[75] Martin RL (2003) J. Chem. Phys. 118: 4775.

[76] Plasser F, Thomitzni B, Bäppler SA, Wenzel J, Rehn DR, Wormit M, Dreuw A (2015) J. Comput. Chem. 36: 1609.

[77] Guido CA, Cortona P, Mennucci B, Adamo C (2013) J. Chem. Theory Comput. 9: 3118.

[78] Peach MJG, Benfield P, Helgaker T, Tozer DJ (2008) J. Chem. Phys. 128: 044118 .

[79] Le Bahers T, Adamo C, Ciofini I (2011) J. Chem. Theory Comput. 7: 2498.

[80] Ciofini I, Le Bahers T, Adamo C, Odobel F, Jacquemin D (2012) J. Phys. Chem. C 116: 11946.

[81] Etienne T, Assfeld X, Monari A (2014) J. Chem. Theory Comput. 10: 3896. 
[82] Etienne T, Assfeld X, Monari A (2014) J. Chem. Theory Comput. 10: 3906.

[83] Moore B, Sun H, Govind N, Kowalski K, Autschbach J (2015) J. Chem. Theory Comput. 11: 3305.

[84] Guido CA, Cortona P, Adamo C (2014) J. Chem. Phys. 140: 104101.

[85] Jacquemin D, Whatelet V, Perpète EA, Adamo C (2009) J. Chem. Theory Comput. 5: 2420.

[86] Adamo C, Jacquemin D (2013) Chem. Soc. Rev. 42: 845.

[87] Kasha M (1959) Rev. Mod. Phys. 31: 162.

[88] Dreuw A, Head-Gordon M (2004) J. Am. Chem. Soc. 126: 4007.

[89] Barford W, Electronic and Optical Properties of Conjugated Polymers, Oxford University Press, New York, 2005.

[90] Gierchner J, Park SY (2013) J. Mater. Chem. C 1: 5818.

[91] Gierchner J, Lüer L, Milián-Medina B, Oelkrug D, Egelhaaf HJ (2013) J. Phys. Chem. Lett. 4: 2686.

[92] Gierchner J, Ehni M, Egelhaaf HJ, Milián-Medina B, Beljonne D, Benmansour H, Bazán GC (2005) J. Chem. Phys. 123: 144914.

[93] Dreuw A, Head-Gordon M (2005) Chem. Rev. 105: 4009. 
- Table 1. Composition of the parameter-free functionals used in this study.

- Table 2. Calculated vertical (lowest) singlet excitation energies (in $\mathrm{eV}$ ) of compounds $[6-12] \mathrm{CPP}$, with different functionals and the 6$31 \mathrm{G}^{*}$ basis set. The oscillator strength $(f)$ values are reported between parentheses.

- Table 3. Nature of the lowest singlet excited-states in terms of occupied $\phi_{i}^{\prime}$ and unoccupied $\phi_{a}^{\prime}$ Natural Transition Orbitals, together with their corresponding occupation number $\kappa_{i a}$ (the threshold for including these occupation numbers is fixed here at 0.01) obtained at the TDA-BE0/6-31G* level.

- Table 4. Calculated vertical (lowest) singlet excitation energies (in eV) of compound $[6] \mathrm{CPP}$ for the two relevant dimer configurations, with different functionals and the $6-31 \mathrm{G}^{*}$ basis set. The oscillator strength $(f)$ values are reported between parentheses. 
Table 1:

\begin{tabular}{llllll}
\hline Type & Name & $\lambda_{x}$ & $\lambda_{c}$ & Exchange & Correlation \\
\hline Hybrid & PBE0 & $1 / 4$ & - & PBE & PBE \\
& PBE0-1/3 & $1 / 3$ & - & PBE & PBE \\
Double-hybrid & PBE0-DH & $1 / 2$ & $1 / 8$ & $\mathrm{PBE}$ & $\mathrm{PBE}$ \\
& PBE-QIDH & $3^{-1 / 3}$ & $1 / 3$ & $\mathrm{PBE}$ & $\mathrm{PBE}$ \\
\hline
\end{tabular}


Table 2:

\begin{tabular}{|c|c|c|c|c|}
\hline Compound & Functional & $\Omega^{\prime}\left(S_{1}\right)$ & $\Omega^{\prime}\left(S_{2}\right)$ & $\Omega^{\prime}\left(S_{3}\right)$ \\
\hline \multirow[t]{5}{*}[6]{$\mathrm{CPP}$} & PBE0 & $2.72(0.0000)$ & $3.75(0.0029)$ & $3.75(0.0028)$ \\
\hline & PBE0-1/3 & $2.88(0.0000)$ & $4.05(0.0234)$ & $4.05(0.0224)$ \\
\hline & PBE0-DH & $3.04(0.0000)$ & $4.27(1.0682)$ & $4.27(1.0710)$ \\
\hline & PBE-QIDH & $3.25(0.0000)$ & $4.41(1.1302)$ & $4.41(1.1285)$ \\
\hline & $\omega \mathrm{B} 97 \mathrm{X}$ & $3.51(0.0000)$ & $4.58(0.9904)$ & $4.58(0.9941)$ \\
\hline \multirow[t]{5}{*}[7]{$\mathrm{CPP}$} & PBE0 & $2.82(0.0136)$ & $3.67(0.0000)$ & $3.75(0.0043)$ \\
\hline & PBE0-1/3 & $2.99(0.0170)$ & $3.99(0.0024)$ & $4.06(1.5868)$ \\
\hline & PBE0-DH & $3.16(0.0235)$ & $4.16(1.6237)$ & $4.24(1.5760)$ \\
\hline & PBE-QIDH & $3.37(0.0308)$ & $4.30(1.5705)$ & $4.39(1.5487)$ \\
\hline & $\omega \mathrm{B} 97 \mathrm{X}$ & $3.62(0.0329)$ & $4.48(1.6145)$ & $4.48(1.5635)$ \\
\hline \multirow[t]{5}{*}{ [8]CPP } & PBE0 & $3.08(0.0000)$ & $3.82(0.0071)$ & $3.82(0.0071)$ \\
\hline & PBE0-1/3 & $3.26(0.0000)$ & $4.08(1.8323)$ & $4.08(1.8323)$ \\
\hline & PBE0-DH & $3.45(0.0000)$ & $4.20(1.9103)$ & $4.20(1.9103)$ \\
\hline & PBE-QIDH & $3.66(0.0000)$ & 4.37 (1.8808) & $4.37(1.8808)$ \\
\hline & $\omega \mathrm{B} 97 \mathrm{X}$ & $3.89(0.0000)$ & $4.57(1.9471)$ & $4.57(1.9471)$ \\
\hline \multirow[t]{5}{*}{ [9]CPP } & PBE0 & $3.11(0.0231)$ & $3.75(0.0448)$ & $3.80(0.2310)$ \\
\hline & PBE0-1/3 & $3.29(0.0302)$ & $3.96(2.2257)$ & $4.02(2.0770)$ \\
\hline & PBE0-DH & $3.48(0.0416)$ & $4.10(2.3036)$ & $4.16(2.2388)$ \\
\hline & PBE-QIDH & $3.69(0.0519)$ & $4.27(2.2451)$ & $4.35(2.2269)$ \\
\hline & $\omega \mathrm{B} 97 \mathrm{X}$ & $3.90(0.0530)$ & $4.47(2.3561)$ & $4.55(2.3512)$ \\
\hline \multirow[t]{5}{*}[10]{$\mathrm{CPP}$} & PBE0 & $3.26(0.0000)$ & $3.82(2.0690)$ & $3.82(2.0471)$ \\
\hline & PBE0-1/3 & $3.45(0.0000)$ & $3.99(2.4345)$ & $4.00(2.4266)$ \\
\hline & PBE0-DH & $3.63(0.0000)$ & $4.14(2.6071)$ & $4.15(2.6037)$ \\
\hline & PBE-QIDH & $3.84(0.0000)$ & $4.33(2.5944)$ & $4.34(2.5936)$ \\
\hline & $\omega \mathrm{B} 97 \mathrm{X}$ & $4.04(0.0000)$ & $4.53(2.7519)$ & $4.53(2.7497)$ \\
\hline
\end{tabular}


Table 2 (cont.):

\begin{tabular}{lllll}
\hline Compound & Functional & $\Omega^{\prime}\left(S_{1}\right)$ & $\Omega^{\prime}\left(S_{2}\right)$ & $\Omega^{\prime}\left(S_{3}\right)$ \\
\hline$[11] \mathrm{CPP}$ & PBE0 & $3.27(0.0309)$ & $3.74(2.4835)$ & $3.77(2.2727)$ \\
& PBE0-1/3 & $3.45(0.0394)$ & $3.91(2.7947)$ & $3.96(2.6670)$ \\
& PBE0-DH & $3.63(0.0502)$ & $4.06(2.9657)$ & $4.12(2.9170)$ \\
& PBE-QIDH & $3.84(0.0593)$ & $4.25(2.9274)$ & $4.31(2.9245)$ \\
& $\omega B 97 X$ & $4.04(0.0582)$ & $4.45(3.1007)$ & $4.45(3.1178)$ \\
\hline 12$] \mathrm{CPP}$ & PBE0 & $3.36(0.0000)$ & $3.76(2.6413)$ & $3.76(2.6410)$ \\
& PBE0-1/3 & $3.55(0.0000)$ & $3.94(3.0322)$ & $3.94(3.0322)$ \\
& PBE0-DH & $3.72(0.0000)$ & $4.10(3.3010)$ & $4.10(3.3010)$ \\
& PBE-QIDH & $3.93(0.0000)$ & $4.30(3.3055)$ & $4.30(3.3055)$ \\
& $\omega B 97 X$ & $4.13(0.0000)$ & $4.50(3.5263)$ & $4.50(3.5263)$ \\
\hline
\end{tabular}


Table 3:

\begin{tabular}{|c|c|c|c|c|c|}
\hline Compound & State & $\phi_{i}^{\prime}$ & $\phi_{a}^{\prime}$ & $\kappa_{i a}$ & $\Omega^{\prime}\left(S_{n}\right)$ \\
\hline \multirow[t]{5}{*}[6]{$\mathrm{CPP}$} & $S_{1}$ & HONTO & LUNTO & 0.9855 & 2.72 \\
\hline & $S_{2}$ & HONTO & LUNTO & 0.5393 & 3.75 \\
\hline & & $(\mathrm{H}-1) \mathrm{ONTO}$ & $(\mathrm{L}+1) \mathrm{UNTO}$ & 0.4536 & \\
\hline & $S_{3}$ & HONTO & LUNTO & 0.5383 & 3.75 \\
\hline & & $(\mathrm{H}-1) \mathrm{ONTO}$ & $(\mathrm{L}+1) \mathrm{UNTO}$ & 0.4546 & \\
\hline
\end{tabular}


Table 4:

\begin{tabular}{|c|c|c|c|c|c|c|c|c|c|c|}
\hline Compound & Configuration & Functional & $\Omega^{\prime}\left(S_{1}\right)$ & $\Omega^{\prime}\left(S_{2}\right)$ & $\Omega^{\prime}\left(S_{3}\right)$ & $\Omega^{\prime}\left(S_{4}\right)$ & $\Omega^{\prime}\left(S_{5}\right)$ & $\Omega^{\prime}\left(S_{6}\right)$ & $\Omega^{\prime}\left(S_{7}\right)$ & $\Omega^{\prime}\left(S_{8}\right)$ \\
\hline \multirow[t]{16}{*}[6]{$\mathrm{CPP}$} & \multirow[t]{8}{*}{ Parallel-like } & \multirow[t]{2}{*}{ PBE0 } & 2.57 & 2.58 & 2.97 & 2.99 & 3.63 & 3.63 & 3.64 & 3.65 \\
\hline & & & $(0.0000)$ & $(0.0000)$ & $(0.0226)$ & $(0.0000)$ & $(0.0011)$ & $(0.0000)$ & $(0.0000)$ & $(0.0019)$ \\
\hline & & \multirow[t]{2}{*}{ PBE0-DH } & 2.89 & 2.90 & 3.66 & 3.67 & 4.10 & 4.14 & 4.21 & 4.22 \\
\hline & & & $(0.0000)$ & $(0.0001)$ & $(0.1654)$ & $(0.0045)$ & $(2.6291)$ & $(0.0000)$ & $(1.9585)$ & $(0.0000)$ \\
\hline & & \multirow[t]{2}{*}{ PBE-QIDH } & 3.09 & 3.10 & 4.21 & 4.23 & 4.24 & 4.26 & 4.35 & 4.35 \\
\hline & & & $(0.0000)$ & $(0.0002)$ & $(0.0130)$ & $(2.8046)$ & $(0.0000)$ & $(0.0000)$ & $(2.1852)$ & $(0.0000)$ \\
\hline & & \multirow[t]{2}{*}{$\omega \mathrm{B} 97 \mathrm{X}$} & 3.37 & 3.38 & 4.41 & 4.45 & 4.53 & 4.53 & 4.60 & 4.61 \\
\hline & & & $(0.0000)$ & $(0.0001)$ & $(2.7526)$ & $(0.0000)$ & $(0.0007)$ & $(1.8557)$ & $(0.0243)$ & $(0.0000)$ \\
\hline & \multirow[t]{8}{*}{ Tubular-like } & \multirow[t]{2}{*}{ PBE0 } & 2.61 & 2.61 & 2.91 & 2.91 & 3.66 & 3.66 & 3.66 & 3.66 \\
\hline & & & $(0.0013)$ & $(0.0013)$ & $(0.0000)$ & $(0.0000)$ & $(0.0059)$ & $(0.0003)$ & $(0.0008)$ & $(0.0011)$ \\
\hline & & \multirow[t]{2}{*}{ PBE0-DH } & 2.92 & 2.92 & 3.53 & 3.53 & 4.09 & 4.10 & 4.23 & 4.26 \\
\hline & & & $(0.0019)$ & $(0.0020)$ & $(0.0000)$ & $(0.0001)$ & $(0.0001)$ & $(0.0001)$ & $(1.6099)$ & $(1.7609)$ \\
\hline & & \multirow[t]{2}{*}{ PBE-QIDH } & 3.12 & 3.13 & 3.81 & 3.81 & 4.23 & 4.23 & 4.39 & 4.40 \\
\hline & & & $(0.0024)$ & $(0.0025)$ & $(0.0010)$ & $(0.0007)$ & $(0.0001)$ & $(0.0001)$ & $(1.9843)$ & $(1.9733)$ \\
\hline & & \multirow[t]{2}{*}{$\omega B 97 X$} & 3.39 & 3.40 & 4.40 & 4.41 & 4.56 & 4.57 & 4.62 & 4.62 \\
\hline & & & $(0.0025)$ & $(0.0028)$ & $(0.0001)$ & $(0.0001)$ & $(1.4300)$ & $(1.6425)$ & $(0.0093)$ & $(0.2186)$ \\
\hline
\end{tabular}


- Figure 1. Chemical structure of the investigated $[n] \mathrm{CPP}$ compounds.

- Figure 2. Evolution of the (lowest) excitation energies of compound [6] CPP, as a function of the exact-like exchange weight $\left(\lambda_{x}\right)$ for the PBE- (top) or BLYP-based (bottom) family of functionals. Note that for the double-hybrid cases (i.e. PBE0-DH and PBE-QIDH or B2PLYP and B2GP-PLYP) the effect of the (D)-like correction is also shown (dashed line).

- Figure 3. Isocontour plots (isosurfaces of $0.00002 \mathrm{e} / \mathrm{au}^{3}$ ) of the transition density for selected $S_{1}-S_{3}$ excited-states (from left to right) calculated at the TDA-PBE0/6-31G* (top line) and the $\omega$ B97X/6-31G* (bottom line) levels. Red and blue colors represent negative and positive values of the orbital distribution leading to the transition density. Figure created with gOpenMol.

- Figure 4. Isocontour plots (isosurfaces of $0.02 \mathrm{e} / \mathrm{au}^{3}$ ) of the dominant Natural Transition Orbitals pairs, calculated at the TDA-PBE0/6$31 \mathrm{G}^{*}$ level, for selected $S_{1}-S_{3}$ excited-states: HONTO \& LUNTO (top line); (H-1)ONTO \& (L+1)NTO (bottom line). For each pair, the hole (particle) is on the left (right). Figure created with gOpenMol.

- Figure 5. Supramolecular configuration of a dimer or [6]CPP molecules: parallel- (a) or tubular-like (b) orientations found in the crystalline state. 
- Figure 6. Values of $\Delta \mathbf{r}$ (in $\AA$ ) for a set of the lowest singlet excitedstates of each dimer configuration, calculated at the TDA-PBE0-DH/6$31 \mathrm{G}^{*}$ level.

- Figure 7. Isocontour plots (isosurfaces of $0.02 \mathrm{e} / \mathrm{au}^{3}$ ) of the dominant Natural Transition Orbitals pairs, calculated at the TDA-PBE0$\mathrm{DH} / 6-31 \mathrm{G}^{*}$ level, for the $S_{1}$ (left) and $S_{2} / S_{3}$ (right) excited-state in the parallel dimer case. Figure created with gOpenMol. 


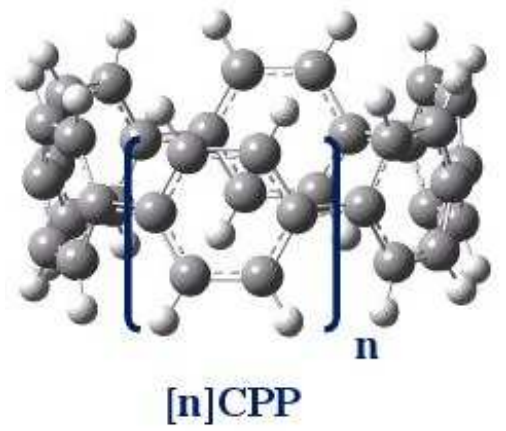

Figure 1. 

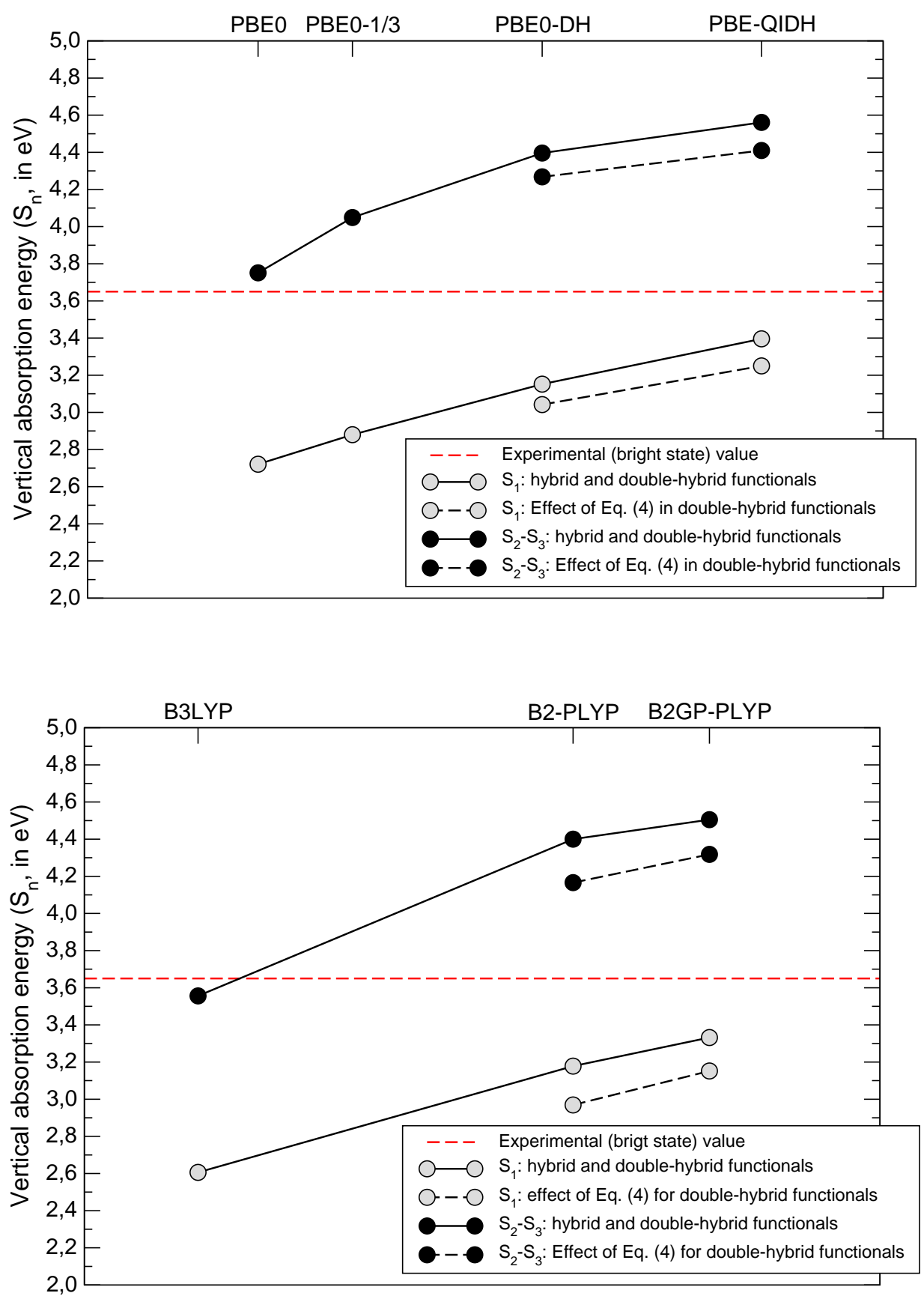

Figure 2. 

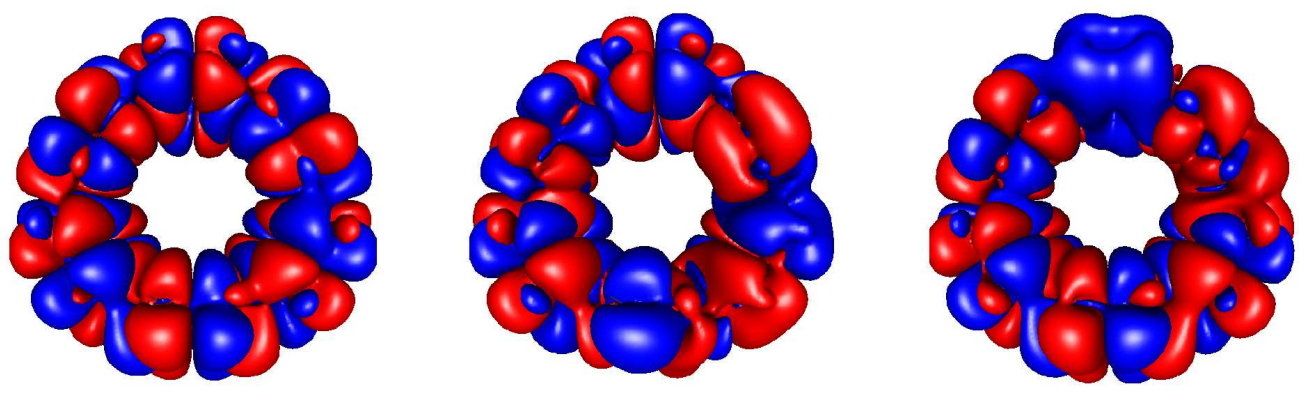

$$
\vec{\mu}_{10}=0
$$

$$
\vec{\mu}_{20} \simeq 0
$$

$\vec{\mu}_{30} \simeq 0$
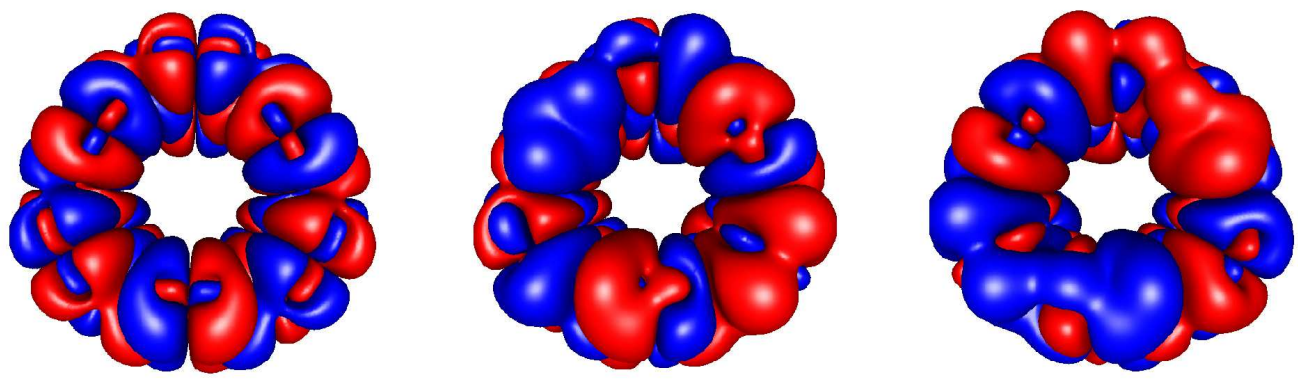

$$
\vec{\mu}_{10}=0
$$

$\vec{\mu}_{20}>>0$

$\vec{\mu}_{30}>>0$

Figure 3. 

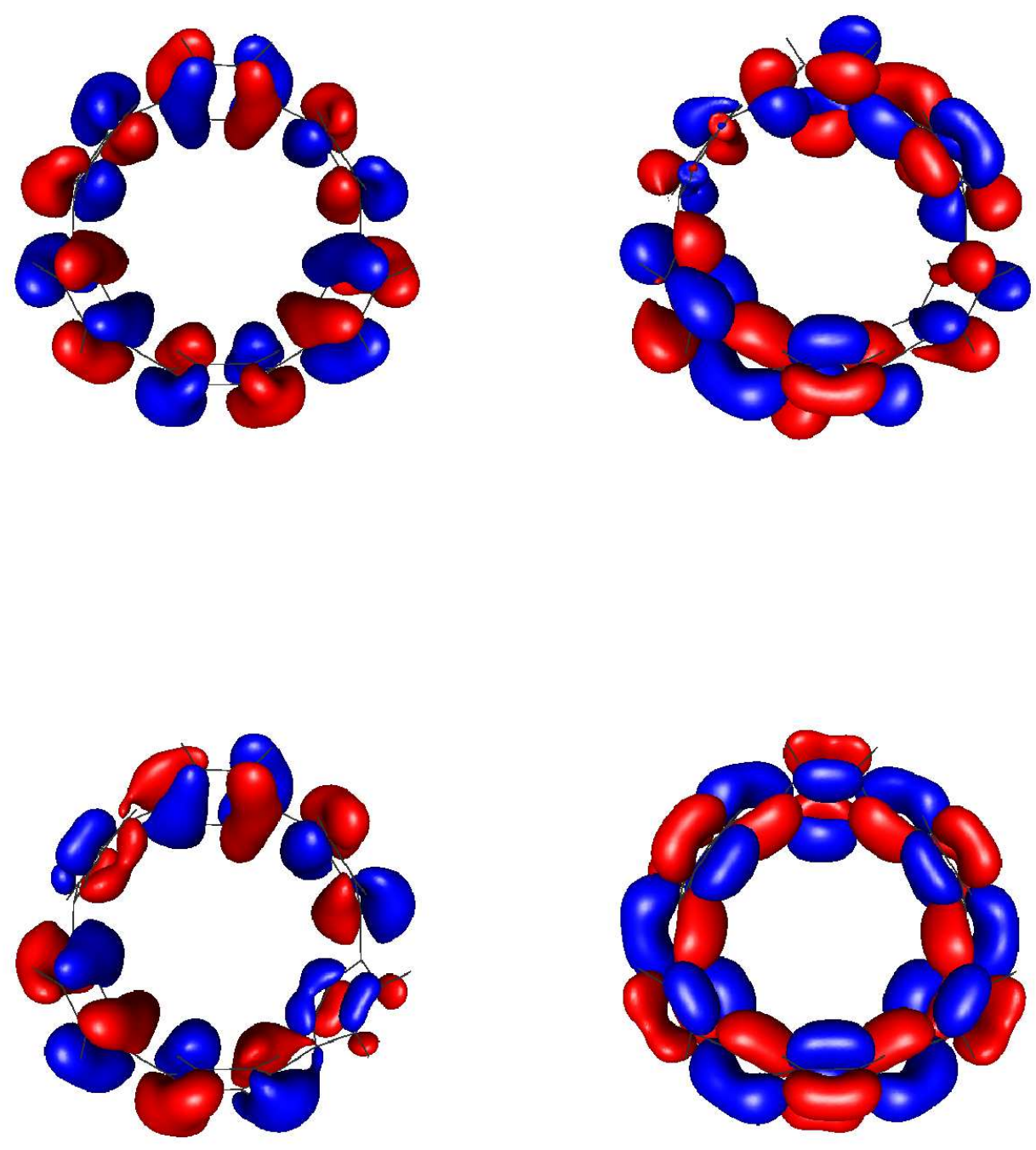

Figure 4 .

38 

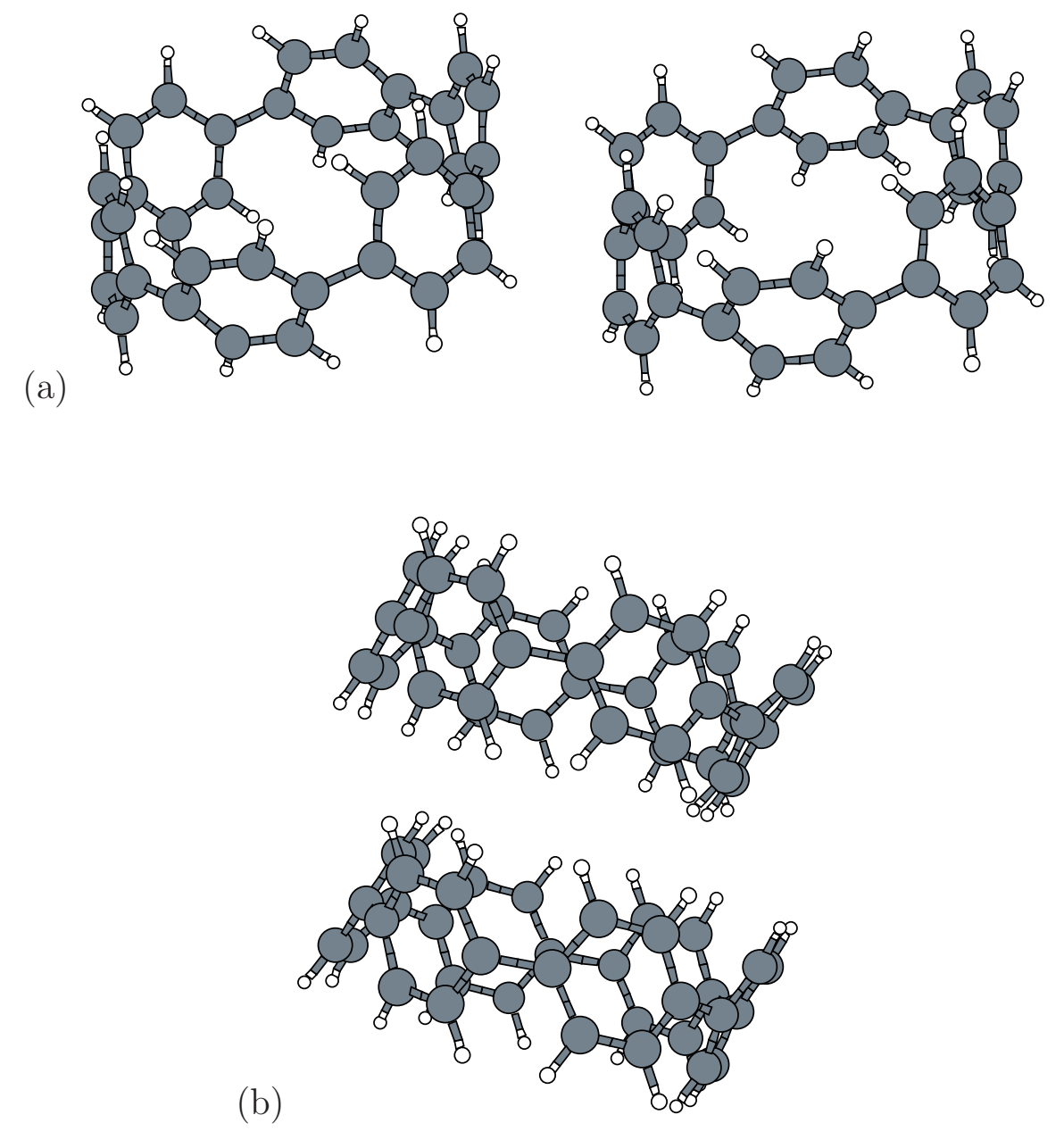

Figure 5. 


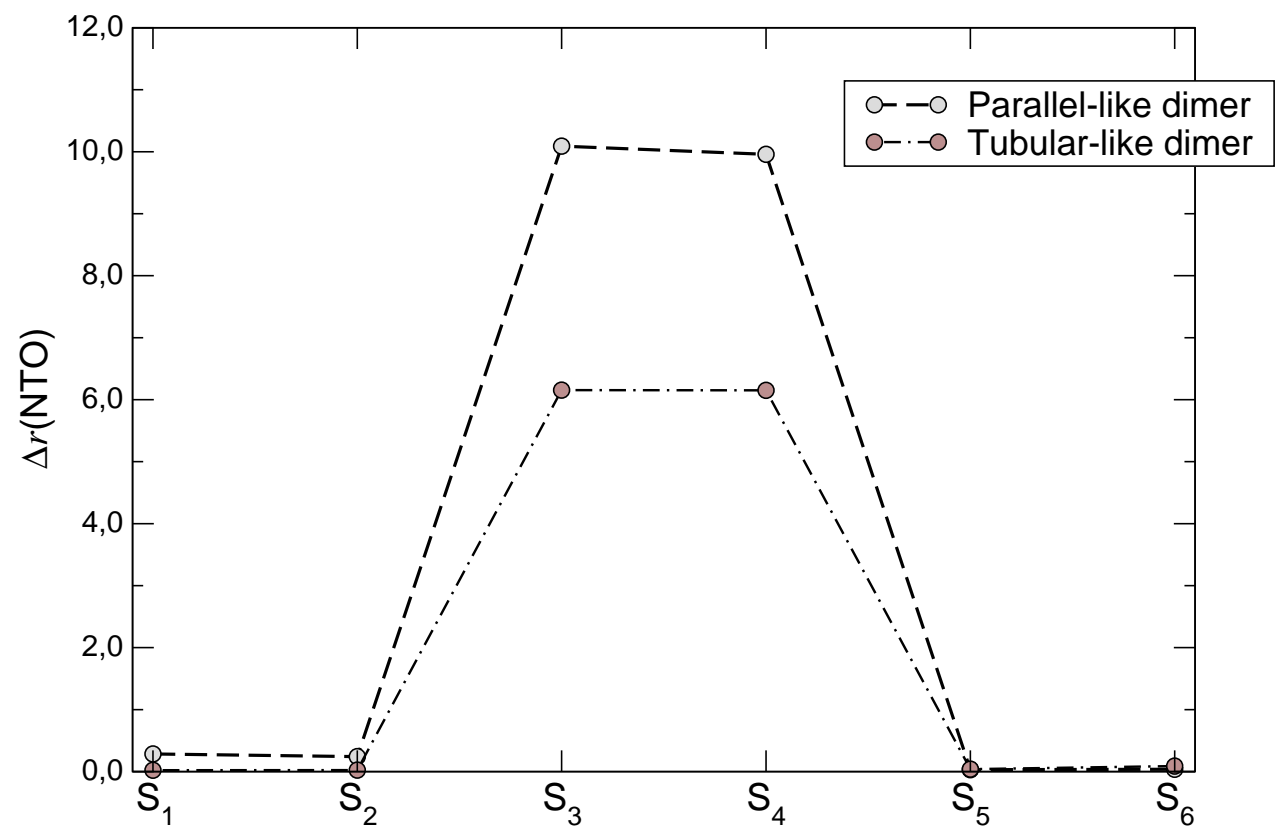

Figure 6. 


\section{W}

HONTO

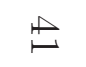

LUNTO

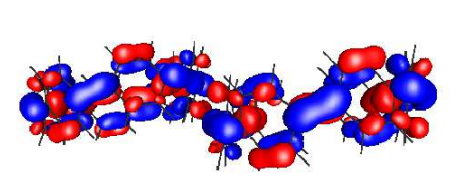

$(\mathrm{L}+1) \mathrm{NTO}$

\section{(1)}

HONTO

Cond

(H-1)NTO

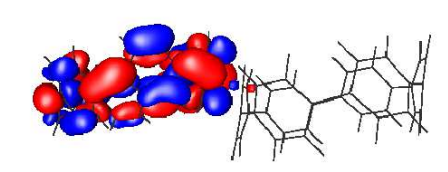

LUNTO

政

$(\mathrm{L}+1) \mathrm{NTO}$

Figure 7. 